\title{
Vacuum Condensates of Dimension Two in Pure Euclidean Yang-Mills
}

\author{
D. Dudal ${ }^{a \dagger \dagger}$, A.R. Fazio ${ }^{\ddagger b}$ V.E.R. Lemes ${ }^{c \S}$, M. Picariello ${ }^{\llbracket d}$, M.S. Sarandy ${ }^{c \|}$, S.P. Sorella ${ }^{c * * \dagger \dagger}$, and H.

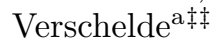 \\ ${ }^{a}$ Ghent University, Department of Mathematical Physics and Astronomy, Krijgslaan 281-S9, B-9000 \\ Gent, Belgium
}

bNational Research Center Demokritos, Ag. Paraskevi, GR-153130 Athens, Hellenic Republic

c UERJ, Universidade do Estado do Rio de Janeiro, Rua São Francisco Xavier 524, 20550-013 Maracanã, Rio de Janeiro, Brazil

${ }^{\mathrm{d}}$ Università degli Studi di Milano, via Celoria, 16, I-20133, Milano, and INFN Milano, Italy

Gluon and ghost condensates of dimension two and their relevance for Yang-Mills theories are briefly reviewed.

\section{The gauge condensate $\left\langle A^{2}\right\rangle$ in the Lan- dau gauge}

\subsection{Motivation}

We shall consider pure Euclidean $S U(N)$ YangMills

$S_{Y M}=-\frac{1}{4} \int d^{4} x F_{\mu \nu}^{a} F_{\mu \nu}^{a}$

In the last few years lattice simulations [1] of the two and three point functions of $S U(N)$ YangMills in the Landau gauge have reported the existence of a large discrepancy between the expected perturbative behavior and the lattice results. The discrepancy is sizeable up to energies $\approx 10 \mathrm{GeV}$, which is a rather big value compared to $\Lambda_{Q C D} \approx(200-300) \mathrm{MeV}$. According to [12], the

\footnotetext{
*Research Assistant of The Fund For Scientific ResearchFlanders, Belgium

${ }^{\dagger}$ david.dudal@rug.ac.be

¥fazio@inp.demokritos.gr

§vitor@dft.if.uerj.br

『marco.picariello@mi.infn.it

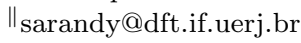

**sorella@uerj.br

${ }^{\dagger}$ Talk given by S.P. Sorella at the International Conference Renormalization Group and Anomalies in Gravity and Cosmology, 17-23 March, 2003, Ouro Preto, MG, Brazil

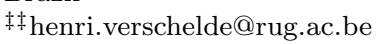

discrepancy could be explained by adding to the perturbative result a power correction of the kind $1 / k^{2}$, by introducing the dimension two gauge condensate $\left\langle A^{2}\right\rangle=\left\langle A_{\mu}^{a} A_{\mu}^{a}\right\rangle$, namely

$$
\begin{aligned}
& k^{2} G^{(2)}\left(k^{2}\right)=G_{\text {PERT }}^{(2)}\left(k^{2}\right)+c \frac{\left\langle A^{2}\right\rangle}{k^{2}} \\
& G^{(2)}=\frac{\delta_{a b}}{3\left(N^{2}-1\right)}\left(\delta_{\mu \nu}-\frac{k_{\mu} k_{\nu}}{k^{2}}\right)\left\langle A_{\mu}^{a}(k) A_{\nu}^{b}(-k)\right\rangle
\end{aligned}
$$

and

$\alpha_{\text {run }}\left(k^{2}\right)=\alpha_{\text {PERT }}\left(k^{2}\right)+c^{\prime} \frac{\left\langle A^{2}\right\rangle}{k^{2}}$

where the coefficients $c, c^{\prime}$ are obtained through OPE [3]. The lattice estimate for the gauge condensate is $\left\langle A^{2}\right\rangle \approx(1.64 G e V)^{2}$ at the energy scale $\mu=10 \mathrm{GeV}$ [1]. The existence of a nonvanishing condensate $\left\langle A^{2}\right\rangle$ could be deeply related to the dynamical mass generation for the gluons and to the instability of the causal perturbative Yang-Mills vacuum [4. Lattice results [5] have indeed reported something like $m_{\text {gluon }} \approx 600 \mathrm{MeV}$. It is worth mentioning that theoretical analysis of the gluon propagator in the Landau gauge have shown that its behavior is suppressed in the infrared region [67891011], in agreement with 
lattice simulations 51213. The gauge condensate $\left\langle A^{2}\right\rangle$ might also be relevant for confinement 14], as it could lead to the area law for the vacuum expectation value of the Wilson loop $W \sim \exp (-\sigma$ Area $)$ with $\sigma \sim\left\langle A^{2}\right\rangle$.

In particular, as underlined in [2], $\left\langle A^{2}\right\rangle$ should receive contributions from both long and short distances, i.e.

$\left\langle A^{2}\right\rangle=\left\langle A^{2}\right\rangle_{\mathrm{LD}}+\left\langle A^{2}\right\rangle_{\mathrm{SD}}$.

For what concerns the long distance part $\left\langle A^{2}\right\rangle_{\mathrm{LD}}$, Ph. Boucaud et al. [15] have established that instantons do contribute to $\left\langle A^{2}\right\rangle_{\mathrm{LD}}$. The lattice estimate of the instanton contribution has been found $\left\langle A^{2}\right\rangle_{\text {INST }} \approx(1.7) \mathrm{GeV}^{2}$. For the short distance contribution $\left\langle A^{2}\right\rangle_{\mathrm{SD}}$, H. Verschelde et al. 16. have been able to obtain the two-loop effective potential for $\left\langle A^{2}\right\rangle$ by combining the Local Composite Operators technique with the Renormalization Group Equations. They obtained a gap equation whose weak coupling solution yields a nonvanishing condensate, resulting in a gluon mass $m_{\text {gluon }} \approx 500 M e V$.

\subsection{Why $A^{2}$ in the Landau gauge}

A simple naive argument shows that $\int d^{4} x A^{2}$ is invariant under infinitesimal gauge transformations in the Landau gauge $\partial A=0$, namely

$\delta A_{\mu}^{a}=-\left(D_{\mu} \omega\right)^{a}$

$\delta \int d^{4} x \frac{1}{2} A^{2}=\int d^{4} x \omega^{a} \partial A^{a}=0$

In the BRST framework we have that, in the Landau gauge, $\int d^{4} x A^{2}$ is BRST invariant on shell

$s \int d^{4} x A^{2}=0+$ eqs. of motion

A more precise meaning for $A^{2}$ is provided by introducing the nonlocal gauge invariant operator $A_{\text {min }}^{2}$, obtained by minimizing $\int d^{4} x A^{2}$ along the gauge orbits, namely

$A_{\min }^{2}=\left[\min _{\{\mathrm{U}\}} \cdot \int d^{4} x\left(A_{\mu}^{U}\right)^{2}\right]$

where $U$ denotes a generic gauge transformation. Of course, $A_{\min }^{2}$ is stationary under gauge transformations. Furthermore, the minimum condition for $\int d^{4} x A^{2}$ is given by the Landau gauge $\partial A=0$. A deep relationship between $\int d^{4} x A^{2}$ and $A_{\min }^{2}$ is thus expected to hold in the Landau gauge. In fact, as discussed in [17, it turns out that in the abelian case $\int d^{4} x A^{2}=A_{\min }^{2}$. Also, from [17, one learns that the condensate $\left\langle A^{2}\right\rangle$ can be used as a useful order parameter for the phase transition of compact $Q E D$ in $3 D$. In the nonabelian case, the situation is more complex. It is true that the Landau gauge condition $\partial A=0$ is a stationary condition for the functional $\int d^{4} x A^{2}$. However, in this case, one has to face the existence of Gribov's ambiguities for large values of the gauge field 7]. A recent discussion about $A_{\min }^{2}$ and Gribov's ambiguities can be found in 18 .

The operator $A^{2}$ displays also remarkable ultraviolet properties. It is multiplicatively renormalizable, its anomalous dimension $\gamma_{A^{2}}$ being available up to three loops in the $\overline{M S}$ scheme [19]. Recently, it has been proven [20] by using BRST Ward identities that $\gamma_{A^{2}}$ is not an independent parameter of the theory,

being expressed as a combination of the gauge beta function $\beta$ and of the anomalous dimension $\gamma_{A}$ of the gauge field $A$, according to the relationship

$\gamma_{A^{2}}=-\left(\frac{\beta(a)}{a}+\gamma_{A}\right), \quad a=\frac{g^{2}}{16 \pi^{2}}$.

\section{Generalization of $\left\langle A^{2}\right\rangle$ to other gauges}

\subsection{The Maximal Abelian Gauge}

The Maximal Abelian gauge (MAG) plays an important role for the dual superconductivity picture for confinement based on the electromagnetic duality proposed by 21]. This gauge is extensively used in lattice simulations. It has provided evidences [22] for the Abelian dominance hypothesis 23] and for monopoles condensation [24]. In the MAG, the gauge field is decomposed according to the generators of the Cartan subgroup of the gauge group. For $S U(2)$

$A_{\mu}^{a} T^{a}=A_{\mu} T^{3}+A_{\mu}^{\alpha} T^{\alpha}, \quad \alpha=1,2$

For the gauge fixing we have

$\int d^{4} x\left[\frac{1}{2 \xi} F^{\alpha} F^{\alpha}-\bar{c}^{\alpha} M^{\alpha \beta} c^{\beta}-g^{2} \xi\left(\bar{c}^{\alpha} \varepsilon^{\alpha \beta} c^{\beta}\right)^{2}\right]$ 
where $\xi$ denotes the gauge parameter and

$F^{\alpha}=D_{\mu}^{\alpha \beta} A_{\mu}^{\beta}=\left(\partial_{\mu} A_{\mu}^{\alpha}+g \varepsilon^{\alpha \beta} A_{\mu} A_{\mu}^{\beta}\right)$

with

$M^{\alpha \beta}=D_{\mu}^{\alpha \gamma} D_{\mu}^{\gamma \beta}+g^{2} \varepsilon^{\alpha \gamma} \varepsilon^{\beta \sigma} A_{\mu}^{\gamma} A_{\mu}^{\sigma}$

The MAG allows for a residual local $U(1)$ invariance, which has to be fixed later on. It is a nonlinear gauge. As a consequence, a quartic ghost interaction has to be introduced for consistency 25. Lattice simulations have shown that the offdiagonal components $A_{\mu}^{\beta}$ acquire a mass [26 27]. These components should decouple at low energies, according to the Abelian dominance. Therefore, the understanding of the mechanism for the dynamical mass generation for the off-diagonal components is fundamental for the Abelian dominance. It is remarkable that the operator $A^{2}$ of the Landau gauge can be generalized to the MAG 2829 . The gluon-ghost dimension two operator

$O_{\mathrm{MAG}}=\left(\frac{1}{2} A_{\mu}^{\alpha} A_{\mu}^{\alpha}+\xi \bar{c}^{\alpha} c^{\alpha}\right)$

has indeed the following property

$s \int d^{4} x O_{\mathrm{MAG}}=0+$ eqs. of motion

The condensate $\left\langle O_{\mathrm{MAG}}\right\rangle$ should play a very important role for the Abelian dominance, as it would provide effective masses for the off-diagonal components. However, at present, very little is known about the operator $O_{\mathrm{MAG}}$ and the possible existence of $\left\langle O_{\mathrm{MAG}}\right\rangle$. Concerning the UV properties of $O_{\mathrm{MAG}}$, it has been proven to be multiplicatively renormalizable 30].

\subsection{The Curci-Ferrari gauge}

The so called Curci-Ferrari gauge resembles very much the MAG. It can thus provide useful insights about the gluon-ghost condensate. For the gauge fixing we have now

$$
\begin{aligned}
\int d^{4} x & \left(\frac{1}{2 \xi}\left(\partial A^{a}\right)^{2}+\bar{c}^{a} \partial_{\mu} D_{\mu} c^{a}+\frac{\xi g}{2} f^{a b c} \partial A^{a} \bar{c}^{b} c^{c}\right. \\
- & \left.\frac{\xi g^{2}}{16} f^{a b c} \bar{c}^{a} \bar{c}^{b} f^{m n c} c^{m} c^{n}\right)
\end{aligned}
$$

where $a=1, \ldots,\left(N^{2}-1\right)$, for $S U(N)$. Notice the presence of the quartic ghost term. The operator

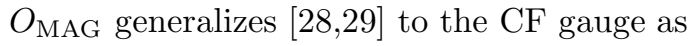

$O_{\mathrm{CF}}=\left(\frac{1}{2} A_{\mu}^{a} A_{\mu}^{a}+\xi \bar{c}^{a} c^{a}\right)$

and

$s \int d^{4} x O_{\mathrm{CF}}=0+$ eqs. of motion

Some properties of the operator $O_{\mathrm{CF}}$ are known. $O_{\mathrm{CF}}$ is multiplicatively renormalizable [29]. Its anomalous dimension has been computed till three loops in the $\overline{M S}$ scheme 19. Recently, the effective potential for $O_{\mathrm{CF}}$ has been obtained in 31, yielding a gap equation whose weak coupling solution gives a nonvanishing condensate $\left\langle O_{\mathrm{CF}}\right\rangle$, resulting in a dynamical mass generation. This gives an indication that something similar should happen in the MAG. It is also worth remarking that the Landau gauge, the MAG and the CF gauge have many features in common. All these gauges possess a larger set of global symmetries, giving rise to the so called Nakanishi-Ojima (NO) algebra 32. The operators $A^{2}, O_{\mathrm{MAG}}, O_{\mathrm{CF}}$ are left invariant ${ }^{10}$ by the NO algebra.

\section{Evidences for ghost condensates}

Contrary to the gauge condensate $\left\langle A^{2}\right\rangle$, the first proposal for the ghost condensation has been made in the Maximal Abelian gauge by 3334 . Due to the quartic ghost-antighost self interaction of the MAG

$\left(\bar{c}^{\alpha} \varepsilon^{\alpha \beta} c^{\beta}\right)^{2}$

ghosts might condense, giving rise to bound states. To some extent, the mechanism is similar to the formation of fermion bound states in the Nambu Jona-Lasinio model. Several channels for the ghost condensates are possible [35, corresponding to different values of the Faddeev-Popov charge, namely

$$
\begin{array}{ll}
\left\langle\bar{c}^{\alpha} \varepsilon^{\alpha \beta} c^{\beta}\right\rangle & \text { Faddeev }- \text { Popov charge } 0 \\
\left\langle c^{\alpha} \varepsilon^{\alpha \beta} c^{\beta}\right\rangle & \text { Faddeev }- \text { Popov charge }+2
\end{array}
$$

${ }^{10}$ Invariant on-shell for what concerns the (anti-)BRST. 
$\left\langle\bar{c}^{\alpha} \varepsilon^{\alpha \beta} \bar{c}^{\beta}\right\rangle \quad$ Faddeev - Popov charge -2

The existence of several channels for the ghost condensation can be related to the dynamical symmetry breaking of the generators of the $S L(2, R)$ subalgebra of the NO algebra 32. It has an interesting analogy with ordinary superconductivity, known as the BCS versus Overhauser effect. The BCS channel corresponds to the charged particle-particle and hole-hole pairing, while the Overhauser to the particle-hole pairing. In the present case the Faddeev-Popov charged condensates $\left\langle c^{\alpha} \varepsilon^{\alpha \beta} c^{\beta}\right\rangle, \quad\left\langle\bar{c}^{\alpha} \varepsilon^{\alpha \beta} \bar{c}^{\beta}\right\rangle$ would correspond to the BCS channel, while $\left\langle\bar{c}^{\alpha} \varepsilon^{\alpha \beta} c^{\beta}\right\rangle$ to the Overhauser channel.

Evidences for the existence of the ghost condensates have been reported also in the CurciFerrari gauge 363738. Although the quartic ghost-antighost interaction is absent in the Landau gauge, it has been possible by combining the Local Composite Operators technique with the Algebraic Renormalization to give evidences for the ghost condensation in this gauge [39.

Many aspects of the gauge and ghost condensation are under investigation [40, deserving a deeper understanding. Some of them are:

- Analysis of the BCS versus Overhauser effect and its relationship with the breaking of the NO algebra, present in MAG, CF and Landau gauge.

- The role of the color and BRST symmetry in the presence of the gauge and ghost condensates.

- Modification of the infrared behavior of the ghost propagator and possible consequences for the Schwinger-Dyson equations. The ghost condensation modifies indeed the off-diagonal ghost propagator in the infrared as

$\left\langle\bar{c}^{\alpha}(k) c^{\beta}(-k)\right\rangle=\frac{k^{2} \delta^{\alpha \beta}+v \varepsilon^{\alpha \beta}}{k^{4}+v^{2}}$

while for the diagonal component

$\left\langle\bar{c}^{3}(k) c^{3}(-k)\right\rangle=\frac{1}{k^{2}}$

where $v$ stands for the value of the condensation. As underlined in 41, both gauge and ghost condensates $\left\langle A^{2}\right\rangle,\left\langle\bar{c}^{\alpha} \varepsilon^{\alpha \beta} c^{\beta}\right\rangle,\left\langle c^{\alpha} \varepsilon^{\alpha \beta} c^{\beta}\right\rangle,\left\langle\bar{c}^{\alpha} \varepsilon^{\alpha \beta} \bar{c}^{\beta}\right\rangle$ might play an important role for a better understanding of the nature of the mass gap in YangMills theories.

\section{Acknowledgments}

S.P. Sorella is grateful to the Organizing Committee of the Conference for the kind invitation and to M. Asorey for many valuable and helpful discussions. The Conselho Nacional de Desenvolvimento Científico e Tecnológico CNPq-Brazil, the Fundação de Amparo a Pesquisa do Estado do Rio de Janeiro (Faperj), the SR2-UERJ and the MIUR-Italy are acknowledged for the financial support. The work of A.R. Fazio was supported by EEC Grant no. HPRN-CT-1999-00161 and partially by Fondazione "Angelo Della Riccia"Ente morale.

\section{REFERENCES}

1. Ph. Boucaud et al. , Phys. Rev. D 60 (1999) 094509; Phys. Rev. D 61 (2000) 114508; Phys. Rev. D 63 (2001) 114003; Phys. Lett. B 493 (2000) 315; JHEP 0004 (2000) 006.

2. F.V. Gubarev and V.I. Zakharov, Phys. Lett. B 501 (2001) 28.

3. Ph. Boucaud, A. Le Yaouanc, J.P. Leroy, J. Micheli, O. Pène, J. Rodríguez-Quintero, Phys.Rev. D 63 (2001) 114003.

4. G.K. Savvidy, Phys. Lett. B 71 (1977) 133.

5. C. Alexandrou, Ph. de Forcrand, E. Follana, Phys. Rev. D 65 (2002) 114508.

6. V. Gribov, Nucl. Phys. B 139 (1978) 1.

7. D. Zwanziger, Nucl. Phys. B 209 (1982) 336;

G. Dell'Antonio and D. Zwanziger, Nucl. Phys. B 326 (1989) 333.

8. R. Alkofer, L. von Smekal, Phys. Rept. 353 (2001) 281.

9. K.-I. Kondo, hep-th/0303251

10. J.C.R. Bloch, hep-ph/0303125

11. D. Zwanziger, hep-th/0206053

12. K. Langfeld, H. Reinhardt, J. Gattnar, Nucl. Phys. B 621 (2002) 131.

13. A. Cucchieri, T. Mendes and A. Taurines, hep-lat/0302022

14. K.-I.Kondo and T. Imai, hep-th/0206173

15. Ph. Boucaud et al, Phys. Rev. D 66 (2002) 034504 .

16. H.Verschelde, K. Knecht, K. Van Acoleyen, M. Vanderkelen, Phys. Lett. B 516 (2001) 307. 
17. F.V. Gubarev, L. Stodolsky and V.I. Zakharov, Phys. Rev. Lett. 86 (2001) 2220.

18. L. Stodolsky, P. Van Baal, V.I. Zakharov, Phys. Lett. B 552 (2003) 214.

19. J. Gracey, Phys. Lett. B 552 (2003) 101.

20. D. Dudal, H. Verschelde, S.P. Sorella, Phys. Lett. B 555 (2003) 126.

21. Y. Nambu, Phys. Rev. D 10 (1974) 4262;

G. 't Hooft, High Energy Physics EPS Int. Conference, Palermo 1975, ed. A. Zichichi; S. Mandelstam, Phys. Rept. 23 (1976) 245.

22. T. Suzuki, I. Yotsuyanagi, Phys. Rev. D 42 (1990) 4257.

23. G. 't Hooft, Nucl. Phys. B 190 (1981) 455;

Z.F. Ezawa and A. Iwazaki, Phys. Rev. D 25 (1982) 2681.

24. A. Kronfeld, G. Schierholz and U.-J. Wiese, Nucl. Phys. B 293 (1987) 461;

A. Kronfeld, M. Laursen, G. Schierholz and U.-J. Wiese, Phys. Lett. B 198 (1987) 516.

25. H. Min, T. Lee and P.Y. Pac, Phys. Rev. D 32 (1985) 440;

A.R. Fazio, V.E.R. Lemes, M.S. Sarandy and S.P. Sorella, Phys. Rev. D64 (2001) 085003;

K.-I. Kondo, T. Shinohara, T. Imai, hep-th/0105268

26. K. Amemiya and H. Suganuma, Phys. Rev. D 60 (1999) 114509.

27. V.G. Bornyakov et al., Phys. Lett. B 559 (2003) 214.

28. K.-I. Kondo, Phys. Lett. B 514 (2001) 335.

29. K.-I. Kondo, T. Murakami, T. Shinohara, T. Imai, Phys. Rev. D 65 (2002) 085034.

30. U. Ellwanger, N. Wschebor, Int. J. Mod. Phys. A 18 (2003) 1595.

31. D. Dudal, H. Verschelde, V. E. R. Lemes, M. S. Sarandy, S. P. Sorella, M. Picariello, hep-th/0302168

32. D. Dudal, H. Verschelde, V.E.R. Lemes, M.S. Sarandy, S.P. Sorella, M. Picariello, JHEP 0212 (2002) 008.

33. M. Schaden, hep-th/9909011, hep-th/0003030 hep-th/0108034.

34. K.-I. Kondo and T. Shinohara, Phys. Lett. B 491 (2000) 263.

35. V.Lemes, M.Sarandy, S.P. Sorella, hep-th/0206251

36. K.-I. Kondo, hep-th/0103141.
37. V.E.R. Lemes, M.S. Sarandy, S.P. Sorella, M. Picariello, A.R. Fazio, Mod. Phys. Lett. A 18 (2003) 711.

38. H. Sawayanagi, Phys Rev. D 67 (2003) 045002.

39. V.E.R. Lemes, M.S. Sarandy and S.P. Sorella, hep-th/0210077, to appear in Ann. Phys.

40. D. Dudal, H. Verschelde, V.E.R. Lemes, M.S. Sarandy, S. P. Sorella, M. Picariello and A. Vicini, work in progress.

41. D. Dudal and H. Verschelde, hep-th/0209025 